GESTÃO DE OPERAÇÃO E LOGÍSTICA 


\title{
MODELAGEM PARA AVALIAÇÃO DE FORNECEDORES DA INDÚSTRIA LATICINISTA
}

\author{
MODELING FOR SUPPLIERS EVALUATION OF DAIRY INDUSTRY
}

Deise Graziele Dickel

Universidade Federal de Santa Maria

Emidio Gressler Teixeira

Universidade Federal de Santa Maria

Gilnei Luiz de Moura

Universidade Federal de Santa Maria

\begin{abstract}
Data de submissão: 22 abr. 20|6. Data de aprovação: 30 nov. 2016. Sistema de avaliação: Double blind review. Universidade FUMEC / FACE. Prof. Dr. Henrique Cordeiro Martins. Prof. Dr. Cid Gonçalves Filho.
\end{abstract}

Adalberto Américo Fischmann

Faculdade de Economia, Administração e Contabilidade da Universidade de São Paulo

\section{RESUMO}

A indústria de alimentos é uma das mais tradicionais estruturas produtivas atuantes no Brasil. No ano de 2009 , representou quase 10\% do PIB e $20 \%$ das exportações totais nacionais. Neste mesmo período, o setor de laticínios estava entre os quatro principais setores desta indústria e chegou a alcançar em $200 \mathrm{I}$ a segunda posição no setor, quando atingiu quase $10 \%$ do faturamento total da indústria de alimentos. $O$ estudo propõe uma modelagem para avaliação de desempenho dos fornecedores deste setor, os quais têm impacto direto na sua produção. Para a realização do estudo, foi utilizada uma análise multicritério, aplicada por meio de uma pesquisa-ação em uma das maiores indústrias de processamento de leite da América Latina, com foco no atendimento aos requisitos dasnormas internacionais de padronização. A modelagem mostrou-se adequada e satisfatória. Os resultados obtidos puderam ser utilizados como base à tomada de decisão.

\section{PALAVRAS-CHAVE}

Avaliação de Fornecedores. Vantagem Competitiva. Setor de Laticínios. Análise Multicritério. Tomada de Decisão. 


\section{ABSTRACT}

The food industry is one of the oldest active productive structures in Brazil. In 2009, it accounted for almost 10\% of GDP and $20 \%$ of total national exports. In the same period, the dairy industry was among the four major sectors of the industry and, arrived in 2001 to reach the second position in the industry, when it reached almost $10 \%$ of the total turnover of the food industry. The study proposes a model for evaluating the performance of suppliers in this sector, which have direct impact on their production. For the study, we used a multi-criteria analysis, applied through an action research in one of the largest milk processing industries in Latin America, focused to satisfy the requirements of international standardization norms. The modeling was adequate and satisfactory. The results could be used for decision-making.

\section{KEYWORDS}

Supplier Evaluation. Competitive Advantage. Dairy Sector. Multi-criteria Analysis. Decision-making.

\section{INTRODUÇÃO}

A indústria brasileira têm expandido seus horizontes nos últimos tempos, principalmente em resposta à competição acirrada no mercado interno e também à globalização. Paralelamente a essas estratégias programadas ou emergentes, é inegável o desenvolvimento e a profissionalização que vem ocorrendo nas empresas estabelecidas no país (BRITO; VASCONCELOS, 2004; CUNHA; SAMANEZ, 20I2). Essa configuração da arena de negócios leva a um novo posicionamento da indústria nacional, que têm exigido que seus players tornem-se cada vez mais competitivos e agressivos ao mercado, tomando a frente em aspectos como o redesenho e otimização de processos, rentabilidade, redução de custos, inovação e o próprio desenvolvimento da cadeia de produção.

A razão do porque as organizações obtêm sucesso ou fracassam é percebida como uma das questões centrais da estra- tégia (PORTER, 2008). Segundo a percepção porteriana, de quase vinte e cinco anos atrás, a criação e exploração de vantagens competitivas é fundamental para o sucesso organizacional. Todavia, as estratégias definidas em um processo de planejamento não garantem por si só o alcance e o sucesso dos objetivos organizacionais, principalmente se considerado a probabilidade da existência de certo gap entre a elaboração e a execução das estratégias (GRAETZ, 2002; MANKINS; STEELE, 2006). Raps (2004), considera que o índice de organizações que têm sucesso na implementação de seus planos estratégicos está no intervalo de $10 \%$ a $30 \%$, o que pode ser visto como fator desestimulador para o uso do planejamento estratégico.

Blumentritt e Danis (2006) apontam que a habilidade de equalizar $o$ ajuste e a coerência entre os fatores competitivos internos e externos - de uma organização é um elemento chave para o sucesso de 
uma administração estratégica, de maneira a facilitar uma alta performance. Deste modo, as macro-estratégias da empresa, atentas à máxima de que o tempo e os recursos disponíveis sempre estão aquém dos desejos e necessidades internos e externos à empresa, podem conduzir a uma tomada de decisão organizacional, que envolva inclusive o design a ser adotado pela estrutura da organização. Neste sentido, um bom resultado é o que se espera como consequência de uma boa decisão. Matheson e Matheson (1998) apontam que a qualidade da tomada de decisão influencia muito as organizações, principalmente no nível estratégico. Genericamente, toda decisão possui quatro pontos de dificuldade: (I) complexidade; (2) incerteza; (3) objetivos múltiplos que se inter-relacionam; e (4) possibilidade de diferentes perspectivas levarem a diferentes conclusões de análise (CLEMEN; REILLY, 200 I).

$O$ tratamento dado a empresa individual também pode ser extendida a um grupo de empresas com estratégias similares, ou mesmo a uma indústria (PORTER, 2008). Neste caso, a indústria nacional brasileira, mais especificamente o setor de alimentação, foi destaque no cenário nacional nestes últimos anos, visto que foi responsável por 9,3\% do Produto Interno Bruto brasileiro em 2009, e 10,2\% em 2014 (ASSOCIAÇÃO BRASILEIRA DAS INDÚSTRIAS DA ALIMENTAÇÃO [ABIA], 20I5). Em termos de faturamento, passou de $\mathrm{R} \$ 291,6$ para $\mathrm{R} \$ 529,6$ bilhões em 2014, e em exportações de $\mathrm{R} \$ 6 \mathrm{I}, 7$ em 2009 para $\mathrm{R} \$ 96,9$ bilhões em 20|4. Dada a relevância do setor e a possibilidade de replicação dos mecanismos de gestão, emerge a necessidade de novas estudos estratégicos nestaarena de negócios, para que então seja possível tomar melhores decisões a respeito de seu posicionamento.

Em um olhar mais incisivo, tem-se que o setor de laticíniostem grande relevância no setor de alimentação. Segundo estudos do United States Department of Agriculture (20I5), verifica-se que o Brasil ocupou a quinta posição no ranking mundial de produção de leite em 20|4, ultrapassado apenas pela União Européia, Índia, Estados Unidos e China. O estado do Rio Grande do Sul, especificamente, deteve $13,3 \%$ do total da produção de laticínios brasileiros em 2014 (INSTITUTO BRASILEIRO DE GEOGRAFIA E ESTATÍSTICA [IBGE], 20।4).

Esse aumento crescente da demanda em termos de quantidade e qualidade, conduz o setor à necessidade da revisão e melhoramento dos seus processos, como por exemplo, o controle do material recebido e o desenvolvimento dos fornecedores que fazem parte desta cadeia e podem impactar diretamente no produto final. Nesse sentido, as atividades administrativas também precisam ser revisitadas com maior profundidade, visando o alinhamento com as demandas do ambiente interno e externo, e.g., implantação de normas internacionais de gestão para padronização destes processos. Desta forma, a avaliação de desempenho dos fornecedores é considerada como um dos fatores mais relevantes para tornar a empresa adequada a essa competição (PORTER, 2008).

$A$ inadequação dos produtos e processos é algo que merece muita atenção. Uma possível diminuição ou parada da produção de uma organização, por exemplo, acarreta inúmeros prejuízos, os quais podem impactar em toda cadeia do setor econômico, visto que a organização torna-se incapaz de manter o fornecimento para as distribuido- 
ras, e o que por consequência, impossibilita atendera demanda do mercado (PORTER, 2008; STADLHOFER, 20 I0). Para garantir esta disponibilidade ao consumidor, dentro da quantidade, da qualidade e dos prazos estabelecidos, as decisões tomadas perante as questões produtivas necessitam ser rápidas, eficazes e balizadas em informações consistentes (JOSHI, NEPAL, RATHORE et al., 20I3).

Deste modo, a fim de atender a crescente necessidade por modelos capazes de mensurar o desempenho de fornecedores e proporcionar alternativas de forma rápida $\mathrm{e}$ eficiente, o que de fato preconiza o ambiente competitivo, é necessário compilar os mais variados critérios para avaliação. Estes modelos devem ser fundamentados no maior número possível de informações relevantes, para assim apoiar efetivamente a tomada de decisão (MARQUES; SILUK, 20I I; OHDAR; RAY, 20I2; GUARNIERI, 20I5).

A partir dessas premissas, este artigo tem como propósito central propor uma modelagem capaz de avaliar o desempenho dos fornecedores críticos do setor de laticínios da indústria alimentícia. Em termos específicos pretende-se: (i) identificar os principais critérios do setor para a determinação de fornecedores críticos para o processo; (ii) relacionar os critérios e subcritérios baseados no atendimento às normas internacionais para a quantificação do desempenho de seus fornecedores; (iii) criar uma modelagem que possa mensurar os critérios e subcritérios levantados; e (iv) aplicar o método em uma indústria de laticínios para validar a modelagem proposta e identificar possíveis melhorias.

A organização analisada neste trabalho é referência em produção de leite em pó na América Latina, e sua capacidade de pro- cessamento é superior a um milhão de litros de leite por dia. A referida empresa está em processo de adequação às cinco normas internacionais de gestão, e por isso foi escolhida para ser o locus de investigação. As normas de padronização que estão sendo implantadas ditam respectivamente sobre qualidade (NBR ISO 900I), meio ambiente (NBR ISO I400I), saúde e segurança ocupacional (OHSAS I800I), segurança alimentar (NBR ISO 22000) e responsabilidade social (NBR ISO I600I), publicadas por órgãos renomados como o ISO, International Organization for Standardization e o BSI, British Standarts Institution.

\section{AVALIAÇÃO DE DESEMPENHO ORGANIZACIONAL}

A função de acompanhamento, mensuração e controle de desempenho é considerada como um dos principais alicerces da gestão estratégica. Portanto, esse controle não pode estar apenas baseado no feedback tradicional, e sim em um processo feedforward das informações, o qual tem possibilidade de compensar a seletividade do planejamento (KHORRAMSHAHGOL, 20I2). Resultados da performance organizacional permitem muitos ganhos, e.g., identificar a distância existente entre a situação atual de uma organização e o nível adotado como excelência, expresso por indicadores oficiais (HILL; JONES, 20I3; KAPLAN; NORTON, 2008; AL-DHAAFRI; YUSOFF; AL-SWIDI, 20I3). Deste modo, como o controle pode ser parametrizado pelos objetivos, metas e estratégias apontadas no planejamento, também pode além de fornecer a sinalização básica do grau de proximidade desses indicadores, acionar medidas corretivas para alcance dos mesmos.

Nas definições primordiais tayloriana e 
fayolina de administração, a função de controle é apresentada como fundamental à métrica e à sincronia das funções planejamento, organização e direção e dinâmica de toda a estrutura organizacional. Todavia, assim como não é possível ao gestor o acesso à todas as possibilidades de ação, também não o é a mensuração de todas as opções, seja pela impossibilidade temporal e física, seja pelo custo de um processo dessa natureza, ou ainda por fatores não mensuráveis, e.g., distribuição de poder em uma organização. Sendo assim, a utilização de indicadores oficiais emerge de modo natural, pela simples circunstância da possibilidade de mensuração de fatores tangíveis e intangíveis, contanto que se adotem métricas bem definidas, rotinas que operacionalizem a coleta de dados e escalas de medição padronizadas, traduzindo-se assim dados dispersos em informações úteis para o gerenciamento de unidades produtivas (GORVINDAN, RAJENDRAN, SARKIS et al., 20I5; OLSON; SLATER, 2002).

Gorton, Angell, Dries et al.(2015) argumentam que a utilização de indicadores exerce função basilar em atividades de planejamento e controle, visto que possibilita o estabelecimento de metas quantificáveis que auxiliam na antecipação de eventos futuros e no acompanhamento dos processos correntes, auxiliando na tomada de decisão e na busca pela excelência operacional. Paralelamente, é indiscutível que a ação de fulfillment passa a parametrizar os indicadores de qualidade de uma organização (LAKHAL, 2009) e exerce papel de fundo na avaliação de desempenho organizacional, em outras palavras, o conceito de atendimento das necessidades e desejos do cliente como principal feedback das ações organizacionais, bem como de sua responsabilidade.

Gomes e Gomes (2014) destacam um ponto importante sobre a avaliação de desempenho, ao elucidar que os resultados esperados podem diferir entre os diversos agentes interessados na atuação de uma organização (shareholders e stakeholders). Notadamente, os proprietários buscam o máximo retorno sobre o investimento (ROI), os empregados visam a máxima remuneração, os clientes anseiam por produtos inovadores de elevada qualidade ao menor preço, o que denota que o objetivo principal muitas vezes é conflitante entre estes players. Dessa forma, é importante definir a quem se destina o Sistema de Medição de Desempenho (SMD) e a qual visão estratégica será alinhado.

Salienta-se ainda, publicações que visam identificar atributos desejáveis em um SMD, como o estudo realizado por Figueiredo, Macedo-Soares, Fuks et al. (2005), que identificou nove atributos geralmente presentes em publicações que tratam sobre SMD: (i) aprendizado organizacional; (ii)análise crítica; (iii) balanceamento; (iv) clareza; (v) dinamismo; (vi) integração; (vii) alinhamento; (viii) participação; e (ix) relacionamento causal. Figueiredo, Macedo-Soares, Fuks et al. (2005)ainda dissertam sobre cada um destes atributos, na tentativa de guiar na escolha por um modelo de avaliação apropriado à situação a ser mensurada. Em consonância, Lemma, Singh e Kaur (20I5) argumentam sobre quatro pontos de vista que devem apoiar a construção de um SMD: (a) transmitir informações fundamentais a respeito do caso, seja com foco econômico ou não; (b) conter rotinas e procedimentos padronizados; (c) promover o cruzamento de informações que permitam a visão sistêmica do negócio, e 
não a representação pontual de dados de processos; e (d) orientar para a melhoria da eficiência e eficácia dos processos, direcionado para as metas.

Sob a luz das características desejáveis para um SMD, destacam-se os Key Performance Indicators (KPIs). Para conceituar KPls e determinar suas especificidades, Parmenter (20I2) enquadra os indicadores em quatro grupos:

a. Key Results Indicators - KRIs (Indicadores Chave de Resultados) - expressam o desempenho atingido em uma perspectiva do Balanced Scorecard (BSC) ou fatores críticos de sucesso;

b. Result Indicators - RIs (Indicadores de Resultado) - expressam um resultado qualquer atingido;

c. Performance Indicators - Pls (Indicadores de Desempenho) - expressam o que deve ser realizado; e

d. Key Performance Indicators KPIs (Indicadores Chave de Desempenho) - expressam o que deve ser realizado para alavancar o desempenho drasticamente.

Conforme supracitado, os KPls são um conjunto de indicadores especiais capazes de refletir de forma quantitativa e condensada o desempenho de um setor específico de uma organização como um todo, atingindo não apenas uma, mas várias perspectivas do BSC ou fatores críticos de sucesso (FCS) (MEYER, 2009; PARMENTER, 20I2; SAMSONOWA, 20I2; MARAFON, ENSSLIN, LACERDA et al., 20I5). Por conseguinte, a utilização de KPls pressupõe que se estabeleça uma estratégia com um alvo que se deseja atingir (KPI objetivo), e por meio do qual se desdobram os FCS, onde a correta identificação desses corresponde, em grande parte, ao êxito na implementação da metodologia (PARMENTER, 20I2; SAMSONOWA, 20I2).

Deste modo, pode-se inferir que a avaliação de desempenho organizacional é essencial nas organizações, podendo influenciar seu nível de sustentabilidade e competitividade. Além disso, os KPls apresentam-se como ferramentas fundamentais para o levantamento das informações necessárias à construção de um SMD adequado.

\section{ADEQUAÇÃO ÀS NORMAS INTERNACIONAIS E AVALIAÇÃO DE FORNECEDORES}

A capacidade das organizações em atender as demandas de seus clientes depende cada vez mais da qualidade dos produtos e serviços ofertados. A busca e manutenção de fornecedores corresponsáveis, comprometidos e bem qualificados, sobretudo, capazes de oferecer suporte às estratégias organizacionais contratantes têm aumentado de forma progressiva e passa ser um considerável diferencial competitivo. As contratantes tornam-se mais seletivas e passam a exigir muito mais dos potenciais fornecedores. Tais exigências são traduzidas na forma de critérios de avaliação, adotados para a comparação entre os diferentes candidatos (VIANA;ALENCAR, 20I2).

Slack, Chambers, Johnston et al. (2013) consideram que dar atenção ao fornecedor é tão importante quanto dar atenção ao cliente. Observa-se que é fundamental que numa cadeia de suprimentos as empresas e seus fornecedores tenham conhecimento de seus pontos fortes e fracos, e em que situação ocorre problemas para que os mesmos sejam sanados. Nessa concepção, - processo de seleção de fornecedores passa a ganhar destaque à medida que se 
torna capaz de escolher os fornecedores que reúnam as características necessárias para a construção de parceria, permitindo dessa forma a redução dos custos de transação (PERUCIA; BALESTRIN; VERSCHOORE, 20l I).

Em outras palavras, o desempenho dos fornecedores passa a exercer papel ainda mais relevante na qualidade dos produtos e serviços acertados com seus contratantes. A agilidade de um fornecedor pode limitar a habilidade de uma organização em responder rapidamente às exigências do mercado (CHRISTOPHER, 2000). Por essa razão, as empresas têm reconhecido a necessidade de selecionar fornecedores competentes para atender devidamente as requisições de seus clientes (VIANA; ALENCAR, 20I2).

A literatura acadêmica apresenta uma infinidade de modelos para a seleção e avaliação de fornecedores, justamente pela importância apresentada (BAYAZIT; KARPAK;YAGGI, 2006; MENDOZA, 2007; CONSTANTINO; PELLEGRINO, 20I0; MELANDER, 20II; MWIKALI; KAVALE, 2012; LADD, 20I3; PARANIKAS, WHITEFORD, TEVELSON et al.,20I5; GUARNIERI, 20I5). Percebida tal importância, as normas de padronização internacional buscam alinhar-se no sentido de incorporar critérios que permitam às organizações avaliarem seus fornecedores e garantirem não somente a qualidade do produto final, mas também o menor impacto ambiental, ocupacional e social.

Enquanto a norma ISO 900I (ASSOCIAÇÃO BRASILEIRA DE NORMAS TÉCNICAS [ABNT], 2008) procura estabelecer uma relação de benefício mútuo entre empresa e fornecedor para garantir a qualidade do produto, a ISO I400I (AS-
SOCIAÇÃO BRASILEIRA DE NORMAS TÉCNICAS [ABNT], 2004a) busca, além da qualidade, o fornecimento com menor impacto ambiental. Por sua vez, a OHSAS I800I (BRITISH STANDARDS INSTITUTION [BSI], 2007) procura selecionar fornecedores com vista a produzir o menor risco ocupacional. Estas três normas podem ser aplicadas a qualquer tipo e porte de organização, embora alguns segmentos específicos ainda possam adotar outras especificações, como o caso da NBR ISO 22000 (ASSOCIAÇÃO BRASILEIRA DE NORMAS TÉCNICAS [ABNT], 2006), a qual tem por propósito principal, garantir a segurança alimentar e, assim, requerer que os fornecedores de insumos estejam também atendendo todas as normas que garantem essa segurança.

A norma ISO I600I (ASSOCIAÇÃO BRASILEIRA DE NORMAS TÉCNICAS [ABNT], 2004b) versa sob as diretrizes relativas à responsabilidade social.A relevância desta norma está relacionada ao crescimento das exigências do consumidor quanto a preocupações éticas e ambientais. A nova geração de consumidores está alinhada à sua conscientização, ou seja, sua decisão de compra está alicerçada não apenas nas informações de que o produto é saboroso ou que sua produção não polui, ou ao menos que se empenha em poluir menos, mas passa a exigir produtos oriundos de uma organização socialmente responsável (ORSATO, 2006; CARVALHO, 20I0).

Tais normas internacionais, além de assegurar qualidade e demais atributos, está intimamente ligada à interncionalização de empresas, uma vez que permite a padronização de preceitos que são adotados nos mais diversos países (VITORELI; CARPINETTI, 20I3). Cada uma das normas, den- 
tro de suas áreas, são estruturadas por requisitos que, uma vez cumpridos, dão aos stakeholders garantias de estar atendendo suas expectativas e necessidades, além de evidenciarem a incorporação de diferentes aspectos de análise no processo seletivo das empresas, nos quais a aplicação vem sendo possibilitada pelo desenvolvimento de métodos que lidem com múltiplos critérios (VIANA; ALENCAR, 20I2).

Dada a importância de tais requisitos normativos, compreende-se que a utilização dos mesmos como critérios de avaliação para fornecedores se torna adequada, bem como satisfaz a visão multicriterial adotada para a presente pesquisa. Sendo assim, os critérios adotados para a modelagem proposta derivam dos requisitos encontrados nas normas internacionais supracitadas, em consonância com o método descrito a seguir.

\section{MÉTODO}

A presente pesquisa pode ser classificada quanto à natureza como qualitativa e quantitativa (LAKATOS; MARCONI, 20I0), caracteriza-se como descritiva (HAIR, BABIN, MONEY et al., 2005), uma vez que busca descrever as características primordiais encontradas em determinada população. A pesquisa classifica-se ainda como pesquisa -ação, uma vez que tem a finalidade de solucionar um problema coletivo, em que o pesquisador e demais atores agem para obter a solução deste problema (GIL, 20l0).

Dentre as diversas metodologias que têm sido desenvolvidas para a construção de modelos de decisão e julgamento, uma das mais recentes vertentes de desenvolvimento metodológico nesse contexto caracteriza-se por se ocupar da solução de problemas decisórios com vários critérios, os quais têm seus pesos pré-estabelecidos de modo a balizar de formal multicriterial a tomada de decisão. Gomes e Gomes (20l4) denominam esta metodologia como análise multicritério.

As Metodologias Multicritério de Apoio a Decisão (Multi Criterial Decision Aid MCDA) contribuem justamente por auxiliar a tornar este processo menos complexo, ao dispor de ferramentas que permitem elucidar as relações de causa e efeito sobre as preferências dos gestores, aumentando o nível de conhecimento sobre o problema, o que difere substancialmente do conceito de busca da solução ótima dos métodos de otimização da Pesquisa Operacional tradicional (BELTON; STEWART, 200I). Ainda, a MCDA não tem por objetivo remover a subjetividade do problema, mas visa torná -la clara aos tomadores de decisão, guiando-os por caminhos lógicos para satisfazer os objetivos de todos os envolvidos (MARAFON, ENSSLIN, LACERDA et al., 20I5).

Com base nestas informações procurouse propor uma metodologia que pudesse avaliar de maneira satisfatória os fornecedores de empresas do ramo de laticínios, dando suporte para decisões relevantes para o desenvolvimento de fornecedores novos e atuais, bem como gerar subsídio para estratégia de fornecedores, colaborando assim para o aumento da competitividade das organizações.

A pesquisa partiu de uma necessidade encontrada pela empresa âncora do estudo, sediada na região centro do Rio Grande do Sul.A escolha dessa empresa deu-se por dois motivos básicos: (i) ser referência em processamento de leite em toda a América Latina, processando mais de um milhão de litros de leite por dia para a produção de leite em pó e subprodutos; e (ii) estar pas- 
sando pelo processo de adequação a cinco normas internacionais de gestão, sendo elas: NBR ISO 900 I (ABNT, 2008), NBR ISO I400I (ABNT, 2004a), OHSAS I800 I (BSI, 2007), NBR ISO 22000 (2006) e NBR ISO I600I (ABNT, 2004b). Este processo veio a colaborar muito no crescimento da gestão empresarial em vários aspectos.

Um dos aspectos levantados neste processo de adequação foi a seleção e a avaliação dos fornecedores, que até então não possuía os processos definidos, nem mesmo uma metodologia uniforme. Partiu-se então desta necessidade da empresa para se propor a modelagem para um sistema de avaliação de desempenho dos fornecedores que atendesse não só a empresa foco, mas todo o setor em que se insere, que pode gerar conhecimento e possivelmente comparação entre os desempenhos de fornecedores das empresas do setor.

No primeiro momento, por meio de reuniões com lideranças da empresa, foi realizado o levantamento das necessidades da organização para possibilitar assim o desenvolvimento da metodologia alinhada com o referencial teórico. A partir destas reuniões e consultas a literatura especializada, construiu-se a seguinte proposta, ilustrada na Figura I.

framework conceitual do Modelo de Avaliação de Fornecedores possui quatro etapas sequenciais como pode ser visualizado na Figura I:

i. Etapa I - Definição de Fatores Críticos - consiste no levantamento geral de todos os fornecedores da organização, sendo que para cada fornecedor identificado é aplicada uma análise de risco sobre o impacto do fornecimento. Apenas os fornecedores que obtiverem alto grau de risco para o bom funcionamento da organização e forem assim considerados críticos, serão avaliados;

ii. Etapa 2 - Definição de Critérios e Subcritérios - constitui o levantamento do conjunto de critérios e subcritérios que serão utilizados como indicadores para a avaliação de cada fornecedor crítico. Esta etapa, como propõe Gil (20l0), é realizada em forma de seminário em que participam os pesquisadores e os profissionais ligados a cadeia de produção da organização em questão e que tenham autoridade e conhecimento suficiente para apontar quais critérios são realmente determinantes para a avaliação dos fornecedores;

iii. Etapa 3 - Atribuição de Pesos aos Critérios e Subcritérios - resume-se na a atribuição dos pesos a cada critério e subcritério, o que permite ser possível determinar a equação matemática responsável por demonstrar o desempenho de cada fornecedor ao final da avaliação. Nesta etapa também participam os profissionais que realizaram a Etapa 2, apontando qual a relevância de cada critério e subcritério dentro do contexto total de critérios; e

iv. Etapa 4 - Aplicação do Modelo - consiste na implementação do modelo mediante o levantamento dos dados necessários e aplicação da equação obtendo-se o índice que indicará o desempenho de cada fornecedor.

Ao final das etapas, serão gerados índices comparativos, os quais podem fomentar informações a empresa, de tal modo que a permita (a) balizar suas decisões re- 


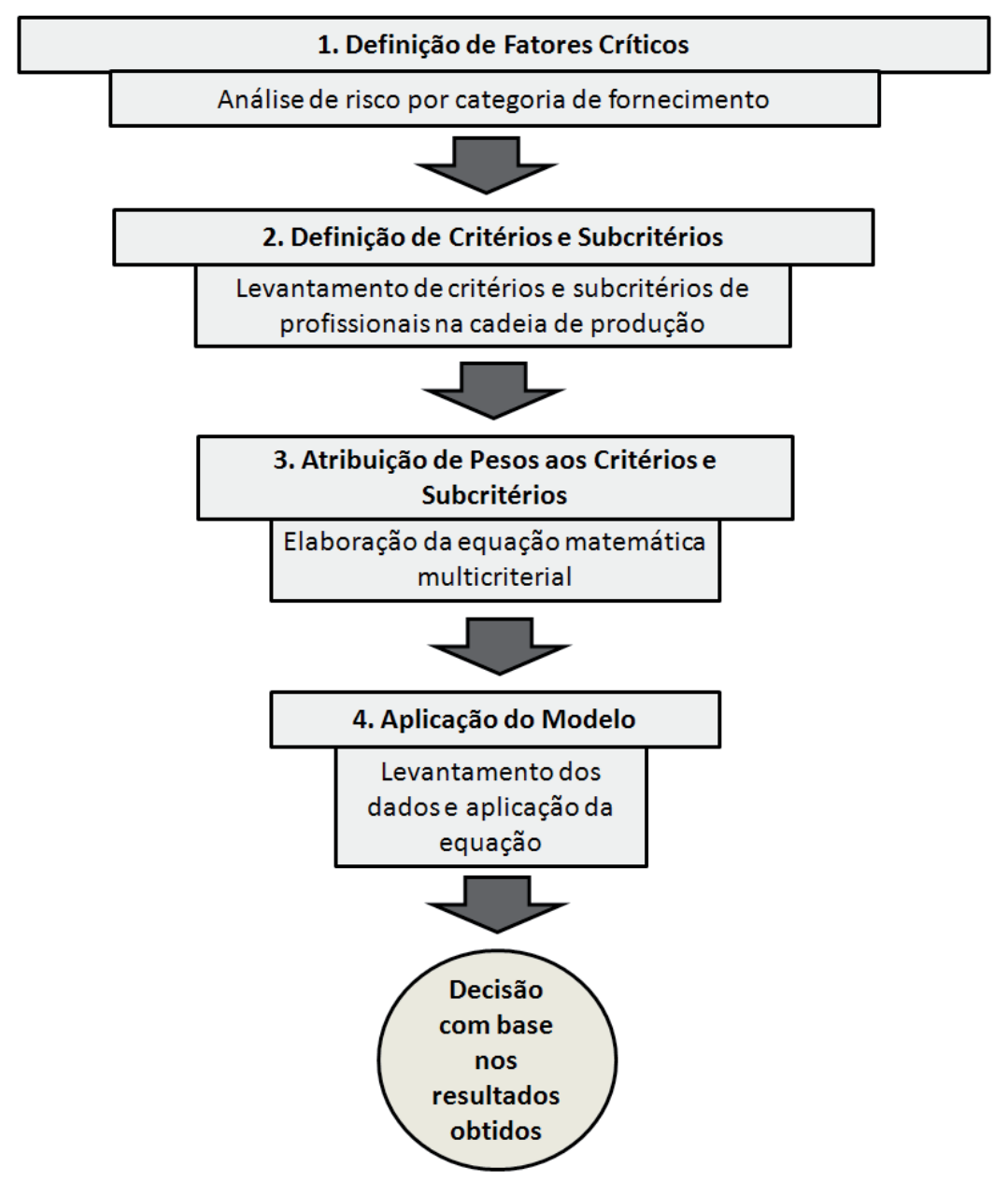

FIGURA 1 - Etapas do Modelo de Avaliação de Fornecedores

Fonte: Elaborado pelos autores

lativas aos fornecedores; (b) gerar o desenvolvimento da sua cadeia de fornecimento; (c) garantir a qualidade dos produtos e serviços adquiridos; e (d) obter alguma significativa vantagem competitiva.

\section{APLICAÇÃO DO MÉTODO E RESULTADOS}

A aplicação do método proposto contemplou o seminário inicial com a empresa escolhida para a pesquisa. Participaram deste seminário os pesquisadores, o diretor superintendente da empresa e as demais lideranças que têm participação nos processos de aquisição, produção, distribuição e áreas de apoio.
Neste seminário foi realizado o levantamento de todos os fornecedores ativos e potenciais da empresa, como também, realizada a decisão sobre o método para categorização, mediante análise de risco, dos fornecedores com maior grau de impacto nos processos do setor de laticínios da indústria de alimentos.

O número total de fornecedores (ativos e potenciais) da empresa foi levantado no cadastro oficial de fornecedores dentro do software que gerencia as operações da empresa. A soma total dos fornecedores levantados no cadastro foi de 147 fornecedores. De posse dessas informações, a partir do conjunto de fatores adotado pela empresa para avaliação 
de risco na escolha de fornecedores (Tabela I), cada um destes fornecedores foi analisado sob a ótica de seis fatores de impacto: (i) Redução de Produção; (ii) Localização do Fornecedor; (iii) Associação de Imagem; (iv) Corresponsabilidade; (v) Influência no Processo; e (vi) Influência no Produto.

A cada um dos seis fatores de impacto ilustrados na Tabela I é atribuída uma pontuação em uma escala que variava de $I$ a 4 , conforme o provável grau de impacto a ser causado. Atribuídas as pontuações dos seis fatores para cada fornecedor listado, realizou-se o cálculo da notação que mensurou o grau de risco de cada fornecedor, sendo que passaram para a segunda fase de avaliação somente os fornecedores cujo resultado da soma dos fatores foi maior ou igual a 14. Este critério foi estabelecido à partir de benchmarking em indústrias do mesmo ramo e acordado entre profissionais especialistas que participaram dos seminários para estabelecimento do método de avaliação.As empresas que atingiram esta pontuação foram enquadrados na categoria de alto risco, como demonstrado na seguinte equação:

\section{$\sum W i \geq 14$}

Onde,

$\mathrm{Wi}$ - representa os fatores de impacto.

Pautando-se nesse parâmetro, obteve-se que do total de 147 empresas fornecedoras analisadas, cinco, agora denominadas "A", "B", “C”, " $D$ " e "E”, obtiveram grau de risco maior que 14 , sendo consideradas de alto risco para a produção e passando para a segunda fase da avaliação, conforme apresenta a Tabela 2.

\section{TABELA 1 - Fatores Avaliados na Análise de Risco na Escolha do Fornecedor}

\begin{tabular}{|c|c|c|}
\hline Fator de impacto & Grau do impacto causado & $\begin{array}{c}\text { Pontuação } \\
\text { Atribuída }\end{array}$ \\
\hline \multirow{4}{*}{$\begin{array}{l}\text { Fator } 1 \text { - Redução } \\
\text { de Produção }\end{array}$} & Não causa redução ou parada na produção & 1 ponto \\
\hline & Pode causar redução da produção em pequeno percentual, no máximo 30\% & 2 pontos \\
\hline & Pode causar redução da produção em grande percentual, mais de $50 \%$ & 3 pontos \\
\hline & Pode causar parada total da produção & 4 pontos \\
\hline \multirow{4}{*}{$\begin{array}{c}\text { Fator } 2 \text { - Localização } \\
\text { do Fornecedor }\end{array}$} & Mesma cidade ou até $160 \mathrm{~km}$, condições de logística sob controle & 1 ponto \\
\hline & Dentro do Estado, condições de logística parcialmente sob controle & 2 pontos \\
\hline & Fora do Estado, condições de logística requerem uma atenção especial & 3 pontos \\
\hline & Outro País, condições de logística totalmente fora de controle & 4 pontos \\
\hline \multirow{3}{*}{$\begin{array}{c}\text { Fator } 3 \text { - Associação } \\
\text { de imagem }\end{array}$} & Falha do fornecedor não impacta na imagem da organização & 1 ponto \\
\hline & Falha do fornecedor causa médio impacto na imagem da organização & 2 pontos \\
\hline & Falha do fornecedor causa alto impacto na imagem da organização & 3 pontos \\
\hline \multirow{3}{*}{ Fator 4 - Corresponsabilidade } & Falha do fornecedor não acarreta corresponsabilidade legal & 1 ponto \\
\hline & Falha do fornecedor acarreta corresponsabilidade legal & 2 pontos \\
\hline & Falha do fornecedor acarreta corresponsabilidade legal com prejuízos financeiros & 3 pontos \\
\hline \multirow{3}{*}{$\begin{array}{l}\text { Fator } 5 \text { - Influência } \\
\text { no Processo }\end{array}$} & Não influencia no processo & 1 ponto \\
\hline & Causa influência de baixo impacto e sob controle & 2 pontos \\
\hline & Causa influência de alto impacto e fora de controle & 3 pontos \\
\hline \multirow{3}{*}{ Fator 6 - Influência no Produto } & Não causa influência no produto final & 1 ponto \\
\hline & Causa influência de baixo impacto e detectável internamente na inspeção & 2 pontos \\
\hline & Causa influência de alto impacto e detectável apenas no cliente & 3 pontos \\
\hline
\end{tabular}

Nota. Fonte: elaborado pelos autores 
TABELA 2 - Resultados de cada Fornecedor na Análise de Risco

\begin{tabular}{c|c|c|c|c|c|c|c}
\hline Fornecedores & Fator 1 & Fator 2 & Fator3 & Fator 4 & Fator 5 & Fator 6 & Risco \\
\hline Empresa A & 4 & 3 & 2 & 3 & 3 & 3 & 18 \\
\hline Empresa B & 4 & 3 & 2 & 2 & 2 & 2 & 15 \\
\hline Empresa C & 4 & 3 & 2 & 3 & 3 & 3 & 18 \\
\hline Empresa D & 3 & 4 & 2 & 3 & 3 & 2 & 17 \\
\hline Empresa E & 3 & 3 & 2 & 2 & 3 & 3 & 16 \\
\hline
\end{tabular}

Nota. Fonte: Elaborado pelos autores.

A segunda etapa foi realizada em forma de seminário pelos pesquisadores juntamente com o diretor superintendente da empresa e as demais gerências responsáveis por áreas influenciadas pelo fornecimento de insumos críticos na produção. Nesta ocasião, foram levantados os principais critérios e também os subcritérios considerados mais importantes para a avaliação do desempenho dos cinco fornecedores críticos. A Figura 2 apresenta os critérios e subcritérios avaliados.

Analisando-se essa etapa, foram identificados três critérios básicos de avaliação dos fornecedores, sendo: $(\mathrm{Cl})$ Critérios Normativos; (C2) Capacidade de Fornecimento; e (C3) Preço. Dentro do critério Cl foram levantados oito subcritérios, sendo: (SCI) Sistema de Qualidade; (SC2) Boas Práticas de Fabricação; (SC3) Controle de Processo; (SC5) Gestão Ambiental; (SC6) Processos Terceirizados; (SC7) Gestão de Saúde e Segurança; e (SC8) Responsabilidade Social/Ética.

Após a definição do sistema de critérios e subcritérios para avaliação, iniciou-se a terceira etapa da metodologia, que consiste na determinação dos pesos relativos a cada um dos critérios e subcritérios com base em documentos e observações realizadas no dia-a-dia da empresa, buscando o atendimento das necessidades da organização para seu bom funcionamento e melhores resultados na relação com for- necedores. Os pesos foram atribuídos aos critérios com números de acordo com a participação relativa, ou seja, os pesos atribuídos a cada critério foi utilizado como taxa de substituição nas equações para cálculo da importância de cada critério numa escala de 0 à $100 \%$, conforme apresenta a Tabela 3.

Para os subcritérios dentro do critério $\mathrm{Cl}$, foram atribuídos pesos de acordo com a percentagem relativa, conforme mostra a Tabela 4.

A geração das taxas de substituição para cada subcritério permitiu se chegar à função de agregação aditiva que calcula o modelo, como demonstrado na seguinte equação:

$$
V(\beta)=\sum_{i=1}^{n} \operatorname{WiVi}(\beta)
$$

Onde:

$V(\beta)$ - corresponde ao valor global da avaliação para cada " $\beta\{\mathrm{A}, \mathrm{B}, \mathrm{C}, \ldots$, $Z$ Z)" para a empresa objeto deste estudo

$W_{i}$ - refere-se às taxas de substituição dos critérios e subcritérios, onde "i $\{1,2, . .$, n $\}$ "

$\mathrm{Vi}(\beta)$ - representa os desempenhos obtidos pela empresa fornecedora nos critérios e subcritérios " $i$ ".

Após o término das três primeiras etapas, chegou-se a etapa final da modelagem 


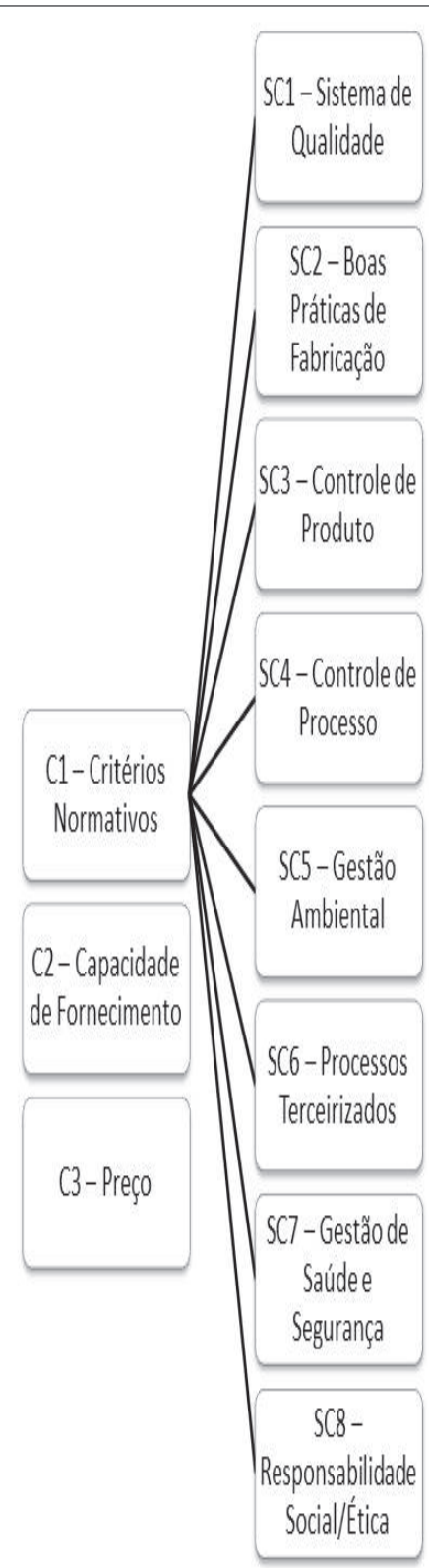

FIGURA 2 - Critérios e Subcritérios Adotados na Avaliação

Fonte: Elaborado pelos autores

que consistiu em implementar o modelo construído. Para isso, primeiramente foi definido em conjunto com a superintendência da empresa, a forma de coleta dos dados para os subcritérios determinados, a qual direcionou a criação do instrumento com base nas normas de referência que a empresa adota, com foco na qualidade, meio ambiente, saúde e segurança ocupa- cional, responsabilidade social e segurança alimentar.

Por meio de auditorias nas plantas industriais dos cinco fornecedores críticos ora identificados, obtiveram-se os dados necessários para calcular o desempenho no critério $\mathrm{Cl}$. Para o cálculo do critério C2 foi utilizada a capacidade de produção do fornecedor comparado com a deman- 
TABELA 3 - Taxas de Substituição Utilizadas para os Critérios

\begin{tabular}{c|c}
\hline Critérios & Taxa de substituição \\
\hline C1 & $50 \%$ \\
\hline C2 & $30 \%$ \\
\hline C3 & $20 \%$ \\
\hline
\end{tabular}

Nota. Fonte: Dados da pesquisa.

TABELA 4 - Taxas de Substituição Utilizadas para os Subcritérios

\begin{tabular}{c|c}
\hline Subcritérios & Taxa de substituição \\
\hline SC1 & $5 \%$ \\
\hline SC2 & $15 \%$ \\
\hline SC3 & $15 \%$ \\
\hline SC4 & $15 \%$ \\
\hline SC5 & $15 \%$ \\
\hline SC6 & $5 \%$ \\
\hline SC7 & $15 \%$ \\
\hline SC8 & $15 \%$ \\
\hline
\end{tabular}

Nota. Fonte: Dados da pesquisa.

da da planta fabril. Assim, para o cálculo do critério C3 utilizaram-se os índices de preço praticado pelos fornecedores no período analisado. Os resultados podem ser acompanhados na Tabela 5.

Realizada a aplicação da modelagem pôde-se observar que o fornecedor "C" obteve o melhor desempenho na avaliação dos fatores de substituição utilizados na função. Mesmo que o desempenho de "C" não tenha obtido os melhores resultados na avaliação individual de cada critério, ao utilizar-se a avaliação multicritério, o referido fornecedor mostrou-se como melhor escolha dentre as outras opções.

Pôde-se observar também que empresa “E” obteve o melhor desempenho no que tange a questão dos critérios normativos, ou seja, o atendimento às normas de referência. No entanto, seu preço apresentou

TABELA 5 - Resultados Obtidos com os Fornecedores Avaliados

\begin{tabular}{c|c|c|c|c|c}
\hline Subcritério & Fornecedor A & Fornecedor B & Fornecedor C & Fornecedor D & Fornecedor E \\
\hline SC1 & 4,3 & 4,3 & 5,0 & 4,3 & 5,0 \\
\hline SC2 & 8,5 & 12,4 & 13,0 & 7,2 & 14,4 \\
\hline SC3 & 13,0 & 12,0 & 14,0 & 12,0 & 12,0 \\
\hline SC4 & 14,0 & 14,0 & 14,0 & 14,0 & 15,0 \\
\hline SC5 & 8,3 & 6,8 & 11,0 & 6,0 & 12,0 \\
\hline SC6 & 3,3 & 3,0 & 3,0 & 3,0 & 5,0 \\
\hline SC7 & 12,0 & 13,5 & 14,0 & 10,5 & 12,8 \\
\hline SC8 & 7,5 & 10,5 & 15,0 & 3,8 & 15,0 \\
\hline Desempenho C1 & 70,90 & 76,39 & 89,05 & 60,62 & 91,15 \\
\hline Desempenho C2 & 82,0 & 71,3 & 77,8 & 92,4 & 84,5 \\
\hline Desempenho C3 & 73,5 & 85,6 & 81,4 & 91,0 & 52,0 \\
\hline Desempenho Global & 74,45 & 76,70 & 84,14 & 76,23 & 81,32 \\
\hline
\end{tabular}

Nota. Fonte: Dados da pesquisa. 
maior desvantagem comparada aos demais fornecedores, o que pode ser indício de que a adoção destes critérios normativos torne o processo mais oneroso para o fornecedor, fazendo com que a empresa tenha a necessidade de repassar os custos ao cliente. Este fenômeno também pode ser explicado pela agregação de valor obtida pelas certificações internacionais, pois uma vez que o cliente está de fato preocupado com a questão do atendimento a estas normas internacionais, pode dispor-se a pagar mais caro pelo produto a ser adquirido.

O pior desempenho foi observado na avaliação do fornecedor "A", que até então era o maior fornecedor em volume. Este desempenho já era esperado pelos participantes da pesquisa, visto que foram registrados diversos desvios na produção devido a falhas neste fornecimento. Esta convicção da organização estudada foi também uma forte motivadora para o início deste trabalho de avaliação dos fornecedores, portanto o método proposto mostrou-se capaz de captar as nuances de desempenho já detectado anteriormente de modo empírico pelos envolvidos.

\section{CONSIDERAÇÕES FINAIS}

$O$ estudo pretendeu encontrar uma forma eficaz de mensurar o desempenho de fornecedores críticos das empresas pertencentes à indústria de laticínios, contemplando os vários critérios envolvidos nesta avaliação, de forma a propor uma modelagem que pudesse ser utilizada para este fim por empresas do setor e que abrangesse o atendimento aos requisitos das normas internacionais de padronização. Para isto, foi realizado um estudo, sob forma de pesquisa-ação, na qual foram identificados os principais critérios da indústria para a de- terminação de fornecedores críticos por meio de uma avaliação de risco.

Neste trabalho, foram relacionados os critérios e subcritérios da indústria de laticínios para a quantificação do desempenho de seus fornecedores por meio de seminários com pesquisadores e colaboradores dos setores envolvidos. Após, elaborou-se uma modelagem para mensurar os critérios e subcritérios identificados e, por fim, esta ferramenta foi aplicada em uma empresa de laticínios para validar o modelo proposto. Os resultados apresentaram cinco fornecedores críticos para a organização, sendo que todos foram avaliados por intermédio de visita técnica conforme a metodologia proposta. Na compilação dos resultados obtidos por cada fornecedor, alcançaram-se resultados bastante próximos, mas com disparidade em cada fator. O fornecedor "C" obteve melhor resultado dentre os fornecedores avaliados, e o pior desempenho foi obtido pelo fornecedor "A", que até a aplicação da modelagem era o maior fornecedor da organização.

Ainda que a modelagem tenha sido aplicada em uma das maiores indústrias de processamento de leite da América Latina, o que é muito pertinente para uma modelagem, ainda precisa de outras aplicações a fim de que possa aglutinar outras singularidades, e.g., Fatores Avaliados na Análise de Risco na Escolha do Fornecedor da empresa escolhida. Esse talvez seja o principal limitador deste estudo.Ademais, a modelagem sugere soluções matemáticas (quantitativas), e geralmente as decisões das empresas dependem de algumas dimensões comportamentais (qualitativas), e.g., motivação, conflito, personalidade, interesses de grupos e particulares, grupos de referência e valores éticos e sociais. (HAYASHI, 200I; 
DAVENPORT, 2005; MACCOBY, 2006; GUARNIERI, 20I5). Soma-se ainda a necessidade de ser fundamental conhecer os players e as driving forces que determinam o comportamento da indústria, uma vez que o desenvolvimento de novos produtos e processos incrementam significativamente a arena competitiva (CLARK;WHEELWRIGHT, 1993).

Deste modo, apesar de suas limitações, este trabalho contribui de modo geral (i) para diminuir a probabilidade de ocorrência de riscos, (ii) para o sucesso da escolha e da manutenção de fornecedores no setor de laticínios da indústria de alimentos. Especificamente, esta pesquisa auxilia para o aprimoramento do processo decisório com relação aos fornecedores da empresa estudada, como também, na adequação do processo de avaliação aos requisitos das normas internacional propostas, de modo a tornar a empresa mais competitiva. 


\section{REFERÊNCIAS}

AL-DHAAFRI, H. S.; YUSOFF, R. Z. B.; AL-SWIDI,A. K.The effect of Total Quality Management, Enterprise Resource Planning and the Entrepreneurial Orientation on the organizational performance: the mediating role of the organizational excellence - a proposed research framework. International Journal of Business Administration, v. 4, n. I, p. 66-85, 2013.

ASSOCIAÇÃO BRASILEIRA DAS INDÚSTRIAS DA ALIMENTAÇÃO. O setor em números. Seção Web. Disponível em: http:// www.abia.org.br/vs/setoremnumeros.aspx. Acesso em: 22dez. 2015.

ASSOCIAÇÃO BRASILEIRA DE NORMAS TÉCNICAS. NBR ISO |400I: Sistema de Gestão Ambiental - Requisitos. Rio de Janeiro:ABNT. 2004a.

ASSOCIAÇÃO BRASILEIRA DE NORMAS TÉCNICAS. NBR ISO |600I: Responsabilidade social - Sistemas da gestão - Requisitos. Rio de Janeiro: ABNT. 2004b.

ASSOCIAÇÃO BRASILEIRA DE NORMAS TÉCNICAS. NBR ISO 22000: Sistemas de gestão da segurança de alimentos - Requisitos para qualquer organização na cadeia produtiva de alimentos. Rio de Janeiro: ABNT. 2006.

ASSOCIAÇÃO BRASILEIRA DE NORMAS TÉCNICAS. NBR ISO 900 I: Sistema de Gestão da Qualidade - Requisitos. Rio de Janeiro:ABNT. 2008.

BAYAZIT, O.; KARPAK, B.; YAGGI, A. A purchasing decision: selecting a supplier for a construction company. Journal of Systems Science and Systems Engineering, v. I5, n. 2, p. 217-23। , 2006.
BELTON, V.; STEWART, T. Multiple criteria decision analysis: an integrated approach. Boston: Springer US, 200I.

BLUMENTRITT, T.; DANIS, W. M. Business strategy types and innovative practices. Journal of Managerial Issues, v. 18, n. 2, p. 274-29I, 2006.

BRITISH STANDARTS INSTITUTION (BSI). OHSAS I800I: Occupational Health and Safety Assessments Series. London: BSI. 2007.

BRITO, L.A. L.; VASCONCELOS, F. C. Performance of Brazilian companies: year effects, line of business and individual firms. Brazilian Administration Review, v. I, n. I, p. I-I5, 2004.

CARVALHO, G. R. (20I0). A indústria de laticínios no Brasil: passado, presente e futuro. Circular Técnica (Embrapa Gado de Leite), v. 102, p. I-I2, 2010.

CHRISTOPHER, M. The agile supply chain: competing in volatile markets. Industrial Marketing Management, v. 29, n. I, p. 37 44, 2000.

CLARK, K. B.; WHEELWRIGHT, S. C. Managing new product and process development: text and cases. New York: Free Press, 1993.

CLEMEN, R. T.; REILLY, T. Making hard decisions with decision tools. Duxbury: Thomson Learning, 200I.

CONSTANTINO, N.; PELLEGRINO,

$R$. Choosing between single and multiple sourcing based on supplier default risk: a real options approach. Journal of Purchasing \& Supply Management, v. I6, n. I, p. 27-40, 2010.

CUNHA, F. A. F. S.; SAMANEZ, C. P. Performance analysis of sustaina- ble investments in the Brazilian stock market: a study about the Corporate Sustainability Index (ISE). Journal of Business Ethics, v. II7, n. I, p. 19-36, 2012.

DAVENPORT, T. H. Thinking for a living: how to get better performance and results from knowledge workers. Boston, Massachusetts: Harvard Business School Press, 2005.

FIGUEIREDO, M. A. D.; MACEDOSOARES, T. D. L. A.; FUKS, S.; FIGUEIREDO, L. C. Definição de atributos desejáveis para auxiliar a auto-avaliação dos novos sistemas de medição de desempenho organizacional. Gestão \& Produção, v. I2, n. 2, p. 305-3 I 5, 2005.

GIL,A.C. Como elaborar projetos de pesquisa. São Paulo: Atlas, 2010.

GOMES, C. F.; GOMES, L.F.A.M. Tomada de decisão gerencial: enfoque multicritério. São Paulo: Atlas, 2014.

GORTON, M.; ANGELL, R.; DRIES, L.; URUTYAN, V.; JACKSON, E.; WHITE, J. Power, buyer trustworthiness and supplier performance: evidence from the Armenian dairy sector. Industrial Marketing Management, v. 50, p. 69-77, 2015.

GORVINDAN, K.; RAJENDRAN, S.; SARKIS, J.; MURUGESAN, P. Multi criteria decision making approaches for green supplier evaluation and selection: a literature review. Journal of Cleaner Production. v. 98, p. 66-83, 2015.

GUARNIERI, P. Synthesis of main criteria, methods and issues of multicriteria supplier selection. Revista de Administração Contemporânea, v. 19, n. I, P. I-25, 2015.

GRAETZ, F. Strategic thinking versus 
strategic planning: towards understanding the complementarities. Management Decision, v. 40, n. 5, p. 456-462, 2002.

HAIR JR., J. F. ; BABIN, B.; MONEY, A. H.; SAMOUEL, P. Fundamentos de métodos de pesquisa em administração. Porto Alegre: Bookman, 2005.

HAYASHI, H. Collective conflict management. Harvard International Review, v. 22, n. 4, p. 82-83, 2001.

HILL, C.W. L.; JONES G. O essencial da administração estratégica - casos reais e aplicação prática da teoria. São Paulo: Saraiva, 2013.

INSTITUTO BRASILEIRO DE GEOGRAFIA E ESTATÍsTICA. Produção da Pecuária Municipal (volume 42). Seção Web. Disponível em: http://www.ibge.gov. br/biblioteca/visualizacao/periodicos/84/ppm_2014_v42_br.pdf. Acesso em: 22dez. 20I5.20I4.

JOSHI, D.; NEPAL, B.; RATHORE,A. P. S.; SHARMA, D. On supply chain competitiveness of Indian automotive component manufacturing industry. International Journal of Production Economics, v. |43, n. I, p.|5I-I6I, 2013.

KAPLAN, R. S.; NORTON, D. P. The execution premium: linking strategy to operations for competitive advantage. Boston: Harvard Business School Press, 2008.

KHORRAMSHAHGOL, R. An integrated strategic approach to supplier evaluation and selection. International Journal of Information Technology \& Decision Making, v.I I, n. I, p. 55-76, 2012.

LADD, SCOTT. E. Supplier sustainability evaluation utilizing multi attribute utility modeling.2013. 63 f. Dissertação
(Mestrado em Engenharia de Produção)- University of Kentucky, Lexington-KY, 2013.

LAKHAL, L. Impact of quality on competitive advantage and organizational performance. Journal of the Operational Research Society, v. 60, n. 5, p. 637-645, 2009.

LAKATOS, E. M.; MARCONI, M. A. Fundamentos de Metodologia Científica. São Paulo: Atlas, 2010.

LEMMA, H. R.; SINGH, R.; KAUR, $N$. Determinants of supply chain coordination of milk and dairy industries in Ethiopia: a case of Addis Ababa and its surroundings. Springer Plus, 4:498, 2015.

MACCOBY, $M$. Is there a best way to lead scientists and engineers? Research Technology Management. v. 49, n. I, p. 60-6I, 2006. MANKINS, M. C.; STEELE, R. Stop making plans, start making decisions. Harvard Business Review, v. 84, n. I, p. 76-84, 2006.

MARAFON, A. D.; ENSSLIN, L.; LACERDA, R. T. O.; ENSSLIN, S.R. The effectiveness of multi-criteria decision aid methodology: a case study of R\&D management. European Journal of Innovation Management, v. 18, n. I, p.86109, 2015.

MARQUES, K. F. S.; SILUK, J. C. M. A gestão da inovação no varejo do RS: um estudo de caso com os empresários da EXPOAGAS 20II. RACE - Revista de Administração, Contabilidade e Economia, v. 10, n. 2, p. 313-336, 2011.

MATHESON, D.; MATHESON, J. The smart organization: creating value through strategic R\&D. Boston, Massachusetts: Harvard Business School Press, 1998.

MELANDER, L. Collaborative new product development: su- pplier selection and purchasing. Linköping - SE: Linköping University Eletronic Press, 20I I.

MENDOZA,A. Effective methodologies for supplier selection and order quantity allocation. 2007. I88 f. Tese (Doutorado em Engenharia Industrial e Pesquisa Operacional)- The Pennsylvania State University, USA, 2007.

MEYER, M.W. Rethinking performance measurement: beyond the balanced scorecard. Cambridge: Cambridge University Press, 2009.

MWIKALI, R.; KAVALE, S. Factors affecting the selection of optimal suppliers in procurement management. International Journal of humanities and social science, v. 2, n. 14, p. I89-193, 2012.

OLSON, E. M.; SLATER, S. F. The balanced scorecard, competitive strategy and performance. Business Horizons, v. 45, n. 3, p. III 7, 2002.

OHDAR, R.; RAY, P. K. Suppliers' performance evaluation and ranking in a supply chain: an analytical hierarchy process-based approach. The IUP Journal of Supply Chain Management, v. IX, n. I, P. 73-90, 2012.

ORSATO, R. J. Competitive environmental strategies: when does it pay to be green? California Management Review, v. 48, n. 2, p. I 27- | 43, 2006.

PARANIKAS, P.; WHITEFORD, G. P.; TEVELSON, B.; BELZ, D. How to negotiate with powerful suppliers. Harvard Business Review, v. 93, n. 7, p. 90-96, 2015.

PARMENTER, D. Key performance indicators for government and nonprofit agencies: implementing winning KPIs. New Jersey: John Wileyand Sons Inc, 2012. PERUCIA, A.; BALESTRIN, A.; VERSCHOORE, J. Coordenação das 
atividades produtivas na indústria brasileira de jogos eletrônicos: hierarquia, mercado ou aliança? Produção, v. 2 I, n. I, p. 64-75, 20 I I.

PORTER, M. E. On competition. Boston: Harvard Business Press, 2008.

RAPS,A. Implementing strategy. Strategic Finance, v. 85 , n. 12, p. 4853, 2004.

SAMSONOWA, T. Industrial research performance management: Key Performance Indicators in the ICT industry. Berlin: Physica-Verlag, 20I 2.
SLACK, N.; CHAMBERS, S.; JOHNSTON, R.; BETTS, A. Gerenciamento de operações e processos: princípios e prática de impacto estratégico. Porto Alegre: Bookman, 2013.

STADLHOFER, G. Corporate real estate performance: contribution to core business competitiveness at global pharmaceutical enterprises. Journal of Corporate Real Estate, v. I2, n. 2, p.96-I I6, 2010. UNITED STATES DEPARTMENT OF AGRICULTURE. Dairy: world markets and trade. Seção
Web. Disponível em: http://apps. fas.usda.gov/psdonline/circulars/ dairy.pdf.Acesso em: 19dez. 2015. 2015.

VIANA, J. C.; ALENCAR, L. H. Metodologias para seleção de fornecedores: uma revisão da literatura. Produção, v. 22, n. 4, p. 625-636, 2012.

VITORELI, G. A.; CARPINETTI, L. C. $R$. Análise da integração dos sistemas de gestão normalizados ISO 9001 e OHSAS 1800I: estudo de casos múltiplos. Gestão e Produção, v. 20, n. I, p. 204-2 I 7, 2013. 